\title{
On Sufficient Degree Conditions for a Graph to be $k$-linked
}

\author{
KEN-ICHI KAW A R A B A Y A S H ${ }^{1}$, \\ ALEXANDR KOSTOCHKA ${ }^{2}$ and GEXIN Y U ${ }^{3 \dagger}$ \\ ${ }^{1}$ Graduate School of Information Sciences (GSIS), Tohoku University, \\ Aramaki aza Aoba 09, Aoba-ku Sendai, Miyagi, 980-8579, Japan \\ (e-mail: k_keniti@dais.is.tohoku.ac.jp) \\ ${ }^{2}$ Department of Mathematics, University of Illinois, Urbana, IL 61801, USA \\ and \\ Institute of Mathematics, Novosibirsk 630090, Russia \\ (e-mail: kostochk@math.uiuc.edu) \\ ${ }^{3}$ Department of Mathematics, University of Illinois, Urbana, IL 61801, USA \\ (e-mail: gexinyu@uiuc.edu)
}

Received 28 November 2003; revised 3 March 2004

\begin{abstract}
A graph is $k$-linked if for every list of $2 k$ vertices $\left\{s_{1}, \ldots, s_{k}, t_{1}, \ldots, t_{k}\right\}$, there exist internally disjoint paths $P_{1}, \ldots, P_{k}$ such that each $P_{i}$ is an $s_{i}, t_{i}$-path. We consider degree conditions and connectivity conditions sufficient to force a graph to be $k$-linked.

Let $D(n, k)$ be the minimum positive integer $d$ such that every $n$-vertex graph with minimum degree at least $d$ is $k$-linked and let $R(n, k)$ be the minimum positive integer $r$ such that every $n$-vertex graph in which the sum of degrees of each pair of non-adjacent vertices is at least $r$ is $k$-linked. The main result of the paper is finding the exact values of $D(n, k)$ and $R(n, k)$ for every $n$ and $k$.

Thomas and Wollan [14] used the bound $D(n, k) \leqslant(n+3 k) / 2-2$ to give sufficient conditions for a graph to be $k$-linked in terms of connectivity. Our bound allows us to modify the Thomas-Wollan proof slightly to show that every $2 k$-connected graph with average degree at least $12 k$ is $k$-linked.
\end{abstract}

\section{Introduction}

Dirac [2] proved that every $n$-vertex graph $G$ with minimum degree at least $n / 2$ is Hamiltonian, and Ore [12] observed that the condition $\delta(G) \geqslant n / 2$ in Dirac's result can be replaced by ' $\sigma_{2}(G) \geqslant n$ ', where $\sigma_{2}(G)$ is the minimum value of the sum $\operatorname{deg}(u)+\operatorname{deg}(v)$ over all pairs $\{u, v\}$ of non-adjacent vertices in $G$.

$\dagger$ This work was partially supported by the NSF grant DMS-0099608 and by the Japan Society for the Promotion of Science for Young Scientists. 
After Chartrand introduced the notion of $k$-ordered graphs, that is, graphs in which for every ordered sequence of $k$ vertices there is a cycle that encounters the vertices of the sequence in the given order, several authors (see, e.g., $[4,11,8,6,5])$ studied the analogue of Dirac's and Ore's sufficient conditions for a graph to be $k$-ordered. Let $D_{0}(n, k)$ denote the minimum positive integer $d$ such that every $n$-vertex graph with minimum degree at least $d$ is $k$-ordered. Similarly, let $R_{0}(n, k)$ denote the minimum positive integer $r$ such that every $n$ vertex graph $G$ with $\sigma_{2}(G) \geqslant r$ is $k$-ordered. Improving on results in [4, 11], it was shown in [6] that $R_{0}(n, k)=n+\lceil(3 k-9) / 2\rceil$ for every $3 \leqslant k \leqslant n / 2$. Furthermore, Kierstead, Sárközy and Selkow [8] showed that $D_{0}(n, k)=\lceil n / 4\rceil+\lfloor k / 2\rfloor-1$ for $3 \leqslant k \leqslant(n+3) / 11$. These bounds demonstrate the interesting phenomenon that $R_{0}(n, k)>2 D_{0}(n, k)$ for $k$ small with respect to $n$.

A graph is $k$-linked if, for every list of $2 k$ vertices $\left\{s_{1}, \ldots, s_{k}, t_{1}, \ldots, t_{k}\right\}$, there exist internally disjoint paths $P_{1}, \ldots, P_{k}$ such that each $P_{i}$ is an $s_{i}, t_{i}$-path. It is a folklore observation that if the number $n$ of vertices of a graph $G$ is at least $2 k$, then in the definition of a $k$-linked graph it is enough to consider only the lists of distinct $s_{1}, \ldots, s_{k}, t_{1}, \ldots, t_{k}$. As in the previous paragraph, let $D(n, k)$ be the minimum positive integer $d$ such that every $n$-vertex graph with minimum degree at least $d$ is $k$-linked. Also, let $R(n, k)$ denote the minimum positive integer $r$ such that every $n$-vertex graph $G$ with $\sigma_{2}(G) \geqslant r$ is $k$ linked. Thomas and Wollan [14] used the bound $D(n, k) \leqslant(n+3 k) / 2-2$ to give sufficient conditions for a graph to be $k$-linked in terms of connectivity. In this paper we determine the exact values of $D(n, k)$ and $R(n, k)$ for all $n$ and $k$.

Theorem 1.1. If $k \geqslant 2$, then

$$
R(n, k)= \begin{cases}2 n-3, & n \leqslant 3 k-1 \\ \left\lfloor\frac{2(n+5 k)}{3}\right\rfloor-3, & 3 k \leqslant n \leqslant 4 k-2 \\ n+2 k-3, & n \geqslant 4 k-1\end{cases}
$$

and

$$
D(n, k)=\left\lceil\frac{R(n, k)}{2}\right\rceil= \begin{cases}n-1, & n \leqslant 3 k-1 \\ \left\lfloor\frac{n+5 k}{3}\right\rfloor-1, & 3 k \leqslant n \leqslant 4 k-2 \\ \left\lceil\frac{n-3}{2}\right\rceil+k, & n \geqslant 4 k-1\end{cases}
$$

Note that $R(3 k, k)<R(3 k-1, k)$. This is the only place for a fixed $k$ where $R(n, k)$ decreases with growing $n$.

Egawa, Faudree, Győri, Ishigami, Schelp and Wang [3] proved the following very closely related result.

Theorem 1.2. ([3]) Let $k \geqslant 2$ and $n \geqslant 3 k$. Let $D_{1}(n, k)$ be the minimum positive integer $d$ such that, for every n-vertex graph $G$ with minimum degree at least $d$ and every matching $M=\left\{s_{i} t_{i} \mid i=1, \ldots, m\right\}$ of size $m \leqslant k$ in $G$, there exist vertex-disjoint cycles $C_{1}, \ldots, C_{m}$ such that $C_{i}$ contains $s_{i} t_{i}$ for each $i=1, \ldots, m$. Similarly, let $R_{1}(n, k)$ be the minimum positive 
integer $r$ such that, for every n-vertex graph $G$ with $\sigma_{2}(G) \geqslant r$ and every matching $M=$ $\left\{s_{i} t_{i} \mid i=1, \ldots, m\right\}$ of size $m \leqslant k$ in $G$, there exist vertex-disjoint cycles $C_{1}, \ldots, C_{m}$ such that $C_{i}$ contains $s_{i} t_{i}$ for each $i=1, \ldots, m$. Then

$$
R_{1}(n, k)= \begin{cases}\left\lfloor\frac{n}{2}\right\rfloor+4 k-2, & 3 k \leqslant n \leqslant 4 k-2, \\ n+2 k-2, & n \geqslant 4 k-1,\end{cases}
$$

and

$$
D_{1}(n, k)= \begin{cases}\left\lceil\frac{n+5 k}{3}\right\rceil-1, & 3 k \leqslant n \leqslant 4 k-2 \\ \left\lceil\frac{n}{2}\right\rceil+k-1, & n \geqslant 4 k-1\end{cases}
$$

This is closely related because, for a graph $G$ and a matching $M=\left\{s_{i} t_{i} \mid i=1, \ldots, m\right\}$, the existence of cycles provided by Theorem 1.2 is equivalent to the existence in $G^{\prime}=G-M$ of vertex-disjoint paths linking $s_{i}$ with $t_{i}$ provided by Theorem 1.1. Although the graphs $G$ and $G^{\prime}$ differ only by a matching, the values of $R(n, k)$ and $R_{1}(n, k)$ for $3 k<n<4 k$ differ significantly. On the other hand, the ideas of the proofs are similar. But neither of the bounds of Theorem 1.2 and Theorem 1.1 can be derived from the other. Also, in terms of linkages, Theorem 1.1 gives slightly better bounds for some parities of $n$ and $k$, which could perhaps be used for extremal problems on linkages.

A very interesting problem is estimating $f(k)$ - the minimum positive integer $f$ such that every $f$-connected graph is $k$-linked. After a series of papers by Jung [7], Larman and Mani [9], Mader [10], and Robertson and Seymour [13], the first linear upper bound for $f$, namely $f(k) \leqslant 22 k$, was proved by Bollobás and Thomason [1]. Very recently, Thomas and Wollan [14] improved this bound to $f(k) \leqslant 16 k$. If one were to use Theorem 1.1 in the Thomas-Wollan proof [14], then their sufficient condition for a graph to be $k$-linked could be relaxed.

Theorem 1.3. Every $2 k$-connected graph $G=(V, E)$ with $|E| \geqslant 6 k|V|$ is $k$-linked. In particular, every $12 k$-connected graph is $k$-linked.

We note that applying Theorem 1.2 also would yield Theorem 1.3. In the next section we prove lower bounds for $D(n, k)$ and $R(n, k)$. Then, in Section 3, the upper bounds are established. In the final section, we show how to modify the Thomas-Wollan proof [14] in order to derive Theorem 1.3.

Using new ideas (in particular, ideas of this paper), Thomas and Wollan improved the upper bound on $f(k)$ further to $f(k) \leqslant 10 k$.

\section{Constructions}

In this section we present examples giving lower bounds for $D(n, k)$ and $R(n, k)$. Consider several cases.

Case 1: $n \leqslant 3 k-1$. Let $G$ be $K_{n}$ with a deleted matching $\left\{\left(v_{1}, v_{2}\right), \ldots,\left(v_{2\lfloor n / 2\rfloor-1}, v_{2\lfloor n / 2\rfloor}\right)\right\}$. Clearly, $\delta(G)=n-2$ and $\sigma_{2}(G)=2 n-4$. Let $m=\min \{k,\lfloor n / 2\rfloor\}$. For $i=1, \ldots, m$, let $s_{i}=v_{2 i-1}$ and $t_{i}=v_{2 i}$. Assume that there exist internally disjoint paths $P_{1}, \ldots, P_{k}$ such 


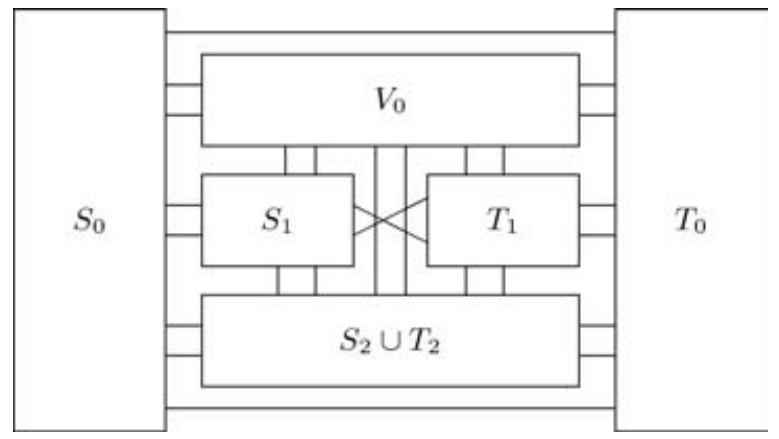

Figure 1. Graph $G$ for Case 2.

that each $P_{i}$ is an $s_{i}, t_{i}$-path. Denote $S=\left\{s_{1}, \ldots, s_{m}, t_{1}, \ldots, t_{m}\right\}$. For every $i$, the path $P_{i}$ contains a vertex $x_{i} \notin S$, since $s_{i} t_{i} \notin E(G)$. Therefore, $n \geqslant|S|+m=3 m$. This is impossible if $n \leqslant 3 k-1, k \geqslant 2$, and $m=\min \{k,\lfloor n / 2\rfloor\}$.

Case 2: $3 k \leqslant n \leqslant 4 k-2$. Let $x=\left\lceil\frac{2 n-5 k}{3}\right\rceil+1$. It is easier to describe the complement $\bar{G}=(V, \bar{E})$ of $G$. Fix six disjoint subsets $S_{0}, S_{1}, S_{2}, T_{0}, T_{1}, T_{2}$ of $V$ with $\left|S_{0}\right|=\left|T_{0}\right|=x-1$, $\left|S_{1}\right|=\left|T_{1}\right|=x$, and $\left|S_{2}\right|=\left|T_{2}\right|=k-x$. Let $V_{0}=V-S_{0}-S_{1}-S_{2}-T_{0}-T_{1}-T_{2}$.

Looking ahead, we may assume that

$$
\begin{array}{ll}
S_{1}=\left\{s_{1}, \ldots, s_{x}\right\}, & S_{2}=\left\{s_{x+1}, \ldots, s_{k}\right\}, \\
T_{1}=\left\{t_{1}, \ldots, t_{x}\right\}, & T_{2}=\left\{t_{x+1}, \ldots, t_{k}\right\} .
\end{array}
$$

The set $\bar{E}$ of edges of $\bar{G}$ is $E_{1} \cup E_{2} \cup E_{3}$, where

$$
\begin{aligned}
E_{1} & =\left\{s_{i} t_{i}: i=1, \ldots, k\right\}, \\
& E_{2}=\left\{v w: v \in S_{0}, w \in T_{1}\right\}, \\
\text { and } \quad & E_{3}=\left\{v w: v \in T_{0}, w \in S_{1}\right\} .
\end{aligned}
$$

The graph $G$ itself is drawn in Figure 1.

Assume that there exist internally disjoint paths $P_{1}, \ldots, P_{k}$ such that each $P_{i}$ is an $s_{i}, t_{i}$-path. As in Case 1, each $P_{i}$ contains a vertex in $S_{0} \cup T_{0} \cup V_{0}$. Moreover, if $i \leqslant x$ (i.e., $s_{i} \in S_{1}$ and $t_{i} \in T_{1}$ ), then either $P_{i}$ contains a vertex in $V_{0}$, or it has at least two internal vertices, since no vertex in $S_{0} \cup T_{0}$ is adjacent to both $s_{i}$ and $t_{i}$. Therefore, $n \geqslant$ $2 k+k+\left(x-\left|V_{0}\right|\right)=5 k-n-2+3 x$. By the definition of $x$, the last expression exceeds $n$, a contradiction.

The vertices in $S_{0} \cup S_{1} \cup T_{0} \cup T_{1}$ have degree $x$ in $\bar{G}$ and all other vertices have degree at most 1. It follows that $\delta(G)=n-1-x=\left\lfloor\frac{n+5 k}{3}\right\rfloor-2$ and $\sigma_{2}(G)=2 n-2-2 x=$ $2\left\lfloor\frac{n+5 k}{3}\right\rfloor-4$. This proves the lower bound on $D(n, k)$ for every $3 k \leqslant n \leqslant 4 k-2$ and on $R(n, k)$ for $3 k \leqslant n \leqslant 4 k-2$ such that $n+5 k \neq 2(\bmod 3)$. For $n+5 k \equiv 2(\bmod 3)$, we slightly modify the construction: we change $x$ to $x=\left\lceil\frac{2 n-5 k+2}{3}\right\rceil$ and move one vertex from $V_{0}$ to $T_{0}$, so that $\left|T_{0}\right|=x$. Then, as in the previous paragraph, we obtain $n \geqslant 2 k+k+$ $\left(x-\left|V_{0}\right|\right)=5 k-n-2+3 x+1$, which contradicts the new definition of $x$. On the other 


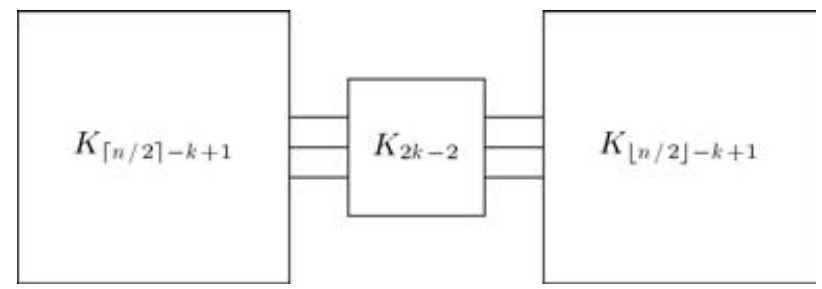

Figure 2. Graph $G$ for Case 3.

hand, $\sigma_{2}(G)=2 n-3-2 x=2\left\lfloor\frac{n+5 k-2}{3}\right\rfloor-3$. Since we have $2\left\lfloor\frac{n+5 k-2}{3}\right\rfloor-3=\left\lfloor\frac{2(n+5 k)}{3}\right\rfloor-4$, for $n+5 k \equiv 2(\bmod 3)$, this completes Case 2 .

Case 3: $n \geqslant 4 k-1$. This is a standard example of a graph with connectivity $2 k-2$ (see Figure 2). Clearly, $\delta(G)=\lfloor n / 2\rfloor+k-2$ and $\sigma_{2}(G)=n+2 k-4$. If $s_{1}, \ldots, s_{k-1}, t_{1}, \ldots, t_{k-1}$ are the vertices of the central $K_{2 k-2}, s_{k}$ belongs to the left $K_{\lceil n / 2\rceil-k+1}$, and $t_{k}$ belongs to the right $K_{\lfloor n / 2\rfloor-k+1}$, then there is no corresponding linkage: there is simply no room for an $s_{k}, t_{k}$-path.

\section{Upper bounds in Theorem 1.1}

Observe that it is enough to prove the upper bound for $R(n, k)$, since $D(n, k) \geqslant R(n, k) / 2$. The case of $n \leqslant 3 k-1$ is obvious, because $\sigma_{2}(G) \geqslant 2 n-3$ means that $G=K_{n}$.

Remark 1. If $n \leqslant 4 k-1$, then $n+2 k-4 \leqslant \frac{2 n+10 k-1}{3}-4$.

Let $n \geqslant 3 k$ and $G=(V, E)$ be a graph on $n$ vertices satisfying the conditions of the theorem. Let $M_{i}=\left\{s_{i}, t_{i}\right\}, i=1, \ldots, k$, be arbitrary disjoint vertex pairs to be linked, and let $M=\cup_{i=1}^{k} M_{i}$. If, say, $s_{k} t_{k} \in E(G)$, then, for the graph $G^{\prime}=G-s_{k}-t_{k}$ with $n^{\prime}=n-2$ vertices and for $k^{\prime}=k-1$, we have $\sigma_{2}\left(G^{\prime}\right) \geqslant R(n, k)-4 \geqslant R\left(n^{\prime}, k^{\prime}\right)$. Therefore, if the theorem holds for $G^{\prime}$, it also holds for $G$. Thus, we may assume that none of the pairs $s_{i} t_{i}$ is an edge in $G$.

We will find the linkage in 3 steps (resembling the steps of the main proof in [6]). On each of the steps, if we cannot perform this step, then $\sigma_{2}(G)<R(n, k)$.

Construct the auxiliary bipartite graph $H$ with partite sets $W_{1}$ and $W_{2}$ as follows. Let $W_{1}=\left\{M_{1}, \ldots, M_{k}\right\}, W_{2}=V-M$, and a pair $\left(M_{i}, v\right)$ be an edge in $H$ if $v \in N_{G}\left(s_{i}\right) \cap N_{G}\left(t_{i}\right)$. If $H$ has a matching saturating $W_{1}$, then this matching gives the required linkage. Otherwise, let $m$ be the size of a maximum matching in $H$.

By the König-Egerváry theorem, there is a $Q \subseteq W_{1}$ with $k-m=|Q|-\left|N_{H}(Q)\right|$. Denote $R=N_{H}(Q)$ and $S=V(G)-M-R$. We may assume that $Q=\left\{M_{i}: i=1, \ldots, q\right\}$. Let $Q^{\prime}=\bigcup_{i=1}^{q}\left\{s_{i}, t_{i}\right\}$ (the elements of $Q$ are pairs, and the elements of $Q^{\prime}$ are all the elements of these pairs). Note that $|Q|=q,\left|Q^{\prime}\right|=2 q,|R|=q-k+m$, and $|S|=n-k-q-m$.

Let $P$ be a maximum matching in $H$. We may assume that only vertices in $D=$ $\left\{M_{1}, \ldots, M_{k-m}\right\}$ are not covered by this matching. Let $D^{\prime}=\left\{s_{i}, t_{i}: 1 \leqslant i \leqslant k-m\right\}$. 
Consider the linkage $\mathscr{P}$ of $M-D$ corresponding to $P$. Let $Z$ be the set of vertices not participating in the linkage.

Lemma 3.1. $n \geqslant 4 k-m$.

Proof. We need to prove this lemma only for $n \leqslant 4 k-1$.

Assume $n \leqslant 4 k-m-1$. Let $M_{i} \in Q$ and $x \in S$. Then either $s_{i}$ or $t_{i}$ is not a neighbour of $x$. Therefore,

$$
\operatorname{deg}\left(s_{i}\right)+\operatorname{deg}\left(t_{i}\right) \leqslant 2(n-2)-|S|=n+k-4+q+m .
$$

For the same reasons, $x$ is not adjacent to at least $q$ vertices in $Q^{\prime}$. We may assume that $x s_{1} \notin E(G)$. If $s_{1} y \notin E(G)$ for every $y \in S$, then

$$
\operatorname{deg}\left(s_{1}\right)+\operatorname{deg}(x) \leqslant(n-2-|S|)+(n-1-q)=n-3+k+m \leqslant n-4+2 k .
$$

By Remark 1, this contradicts (1.1). Otherwise, there is a $y \in S$ with $y s_{1} \in E(G)$ and, therefore, $y t_{1} \notin E(G)$. Thus, by (3.1),

$$
\begin{aligned}
3 \sigma_{2}(G) & \leqslant\left(\operatorname{deg}(x)+\operatorname{deg}\left(s_{1}\right)\right)+\left(\operatorname{deg}(y)+\operatorname{deg}\left(t_{1}\right)\right)+\left(\operatorname{deg}\left(s_{1}\right)+\operatorname{deg}\left(t_{1}\right)\right) \\
& \leqslant 2(n+k-4+q+m)+2(n-q-1) \leqslant 2(n+5 k-6) .
\end{aligned}
$$

It follows that $\sigma_{2}(G)<R(n, k)$.

By Lemma 3.1, for every $i=1, \ldots, k-m$, we can assign a vertex $z_{i} \in Z$ to $s_{i}$ and a vertex $z_{i}^{\prime} \in Z$ to $t_{i}$ so that we assign distinct members of $Z$ to distinct vertices. Also, for every $k-m+1 \leqslant i \leqslant k$, let $y_{i}$ be the common neighbour of $s_{i}$ and $t_{i}$ corresponding to the matching $P$ above. Among such assignments, choose an assignment $\mathscr{A}$ with as many edges $z_{i} s_{i}$ and $z_{i}^{\prime} t_{i}$ as possible. Let $Z^{\prime}=\bigcup_{i=1}^{k-m}\left\{z_{i}, z_{i}^{\prime}\right\}$.

Lemma 3.2. In $\mathscr{A}$, every $z_{i}$ is adjacent to $s_{i}$ and every $z_{i}^{\prime}$ is adjacent to $t_{i}$.

Proof. Assume that $s_{1} z_{1} \notin E(G)$. We will prove that

$$
\left|N\left(s_{1}\right)-M\right| \leqslant\left|M-N\left(z_{1}\right)\right| .
$$

To do this, for every neighbour $w$ of $s_{1}$ outside $M$ we show a non-neighbour $f(w)$ of $z_{1}$ in $M$. First, observe that either $w \in Z^{\prime}$ or $w$ was used in the linkage $\mathscr{P}$, since otherwise we can assign $w$ as $z_{1}$.

Case 1. If $w$ is used in a path $s_{i}, w, t_{i}$ in the linkage $\mathscr{P}$ and $z_{1}$ is adjacent to both $s_{i}$ and $t_{i}$, then by swapping $w$ with $z_{1}$, we will get an assignment with new $z_{1}$ (former $w$ ) adjacent to $s_{1}$. Thus, either $s_{i}$ or $t_{i}$ can be chosen as $f(w)$.

Case 2. If $w \in Z^{\prime}$, say, $w=z_{i}^{\prime}$ (possibly, $i=1$ ), and $z_{1}$ is adjacent to $t_{i}$, then swapping $z_{1}$ with $w$ produces a better assignment. Thus, $z_{1} t_{i} \notin E$, and we let $f(w)=t_{i}$.

Since all $s_{i}$ and $t_{i}$ are disjoint, (3.2) holds, and therefore $\operatorname{deg}\left(s_{1}\right)+\operatorname{deg}\left(z_{1}\right) \leqslant 2(n-2)-$ $|V-M|=n-4+2 k$. By Remark 1 , this yields $\sigma_{2}(G)<R(n, k)$ for each $n \geqslant 3 k$. 
The last step in the proof is given by the next lemma.

Lemma 3.3. The assignment $\mathscr{A}$ in Lemma 3.2 can be chosen in such a way that every $z_{i}$ is adjacent to every $z_{i}^{\prime}$.

Proof. Consider an assignment $\mathscr{A}$ satisfying Lemma 3.2. For $i=1, \ldots, k-m$, let $X_{i}=$ $\left\{s_{i}, t_{i}, z_{i}, z_{i}^{\prime}\right\}$, and for $i=k-m+1, \ldots, k$, let $X_{i}=\left\{s_{i}, t_{i}, y_{i}\right\}$. Let $X=\bigcup_{i=1}^{k} X_{i}$. Choose $\mathscr{A}$ in Lemma 3.2 so that as many as possible $z_{i}$ are adjacent to corresponding $z_{i}^{\prime}$. Suppose that the lemma does not hold. Then we may renumber $\left(s_{i}, t_{i}\right)$ so that $z_{1} z_{1}^{\prime} \notin E(G)$. Let $A=N\left(s_{1}\right) \cap(V(G)-X)$ and $B=N\left(t_{1}\right) \cap(V(G)-X)$.

Note that $N\left(A+z_{1}\right) \cap\left(B+z_{1}^{\prime}\right)=\emptyset$. For $i=1, \ldots, k$, let $k_{i}$ denote the number of neighbours of $X_{1}$ (with multiplicities) in $X_{i}$. Since each member of $X_{1}$ has exactly one neighbour in $X_{1}, k_{1}=4$.

Claim 3.4. If $2 \leqslant i \leqslant k-m$, then $k_{j} \leqslant 12$. If $k-m+1 \leqslant i \leqslant k$, then $k_{j} \leqslant 10$.

\section{Proof.}

Case 1: $2 \leqslant i \leqslant k-m$. By the maximality of $m$, neither of $z_{1}, z_{1}^{\prime}$ is a common neighbour of $s_{i}$ and $t_{i}$ and neither of $z_{i}, z_{i}^{\prime}$ is a common neighbour of $s_{1}$ and $t_{1}$. Therefore, $k_{i} \leqslant$ $\left|X_{1}\right| \cdot\left|X_{i}\right|-4=12$.

Case 2: $k-m+1 \leqslant i \leqslant k$.

Subcase 2.1: $s_{1} y_{i}, t_{1} y_{i} \in E(G)$. If some of $z_{1}, z_{1}^{\prime}$ (say, $z_{1}$ ) is a common neighbour of $s_{i}$ and $t_{i}$, then assigning $z_{1}$ as the new $y_{i}$ and assigning the old $y_{i}$ as the new $y_{1}$ will contradict the maximality of $m$. Otherwise, $k_{i} \leqslant\left|X_{1}\right| \cdot\left|X_{i}\right|-2=3 \cdot 4-2=10$.

Subcase 2.2: $s_{1} y_{i} \notin E(G)$. If all the four edges $z_{1}^{\prime} s_{i}, z_{1}^{\prime} t_{i}, z_{1} y_{i}, t_{1} y_{i}$ are in $E(G)$, then we can swap $y_{i}$ with $z_{1}^{\prime}$ and get a better assignment, since the new $z_{1}^{\prime}$ is adjacent to $z_{1}$. Otherwise, we again have $k_{i} \leqslant 10$.

Claim 3.5. For each $v \notin X,\left|N(v) \cap\left\{s_{1}, t_{1}, z_{1}, z_{1}^{\prime}\right\}\right| \leqslant 2$.

Proof. Otherwise, we can swap $v$ with either $z_{1}$ or $z_{1}^{\prime}$ so that the new assignment is better.

Let $F=\operatorname{deg}\left(s_{1}\right)+\operatorname{deg}\left(t_{1}\right)+\operatorname{deg}\left(z_{1}\right)+\operatorname{deg}\left(z_{1}^{\prime}\right)$. In view of the claims above, $F \leqslant 2(n-$ $|X|)+4+12(k-m-1)+10 m$. Since $|X|=4(k-m)+3 m$, we obtain

$$
F \leqslant 2(n-4 k+m)+4+12(k-m-1)+10 m=2 n+4 k-8 .
$$

Since $F \geqslant 2 \sigma_{2}(G) \geqslant 4 \delta(G)$, we obtain a contradiction to both (1.1) and (1.2). This proves the lemma, and thus the theorem as well.

\section{Connectivity conditions}

Thomas and Wollan [14] showed that $f(k) \leqslant 16 k$ by proving the following (stronger) result. 
Theorem 4.1. ([14]) Every $2 k$-connected graph $G$ with $|E(G)| \geqslant 8 k|V(G)|$ is $k$-linked.

Most of the proof works under weaker restrictions on the average degree. The bottleneck for the bounds in Theorem 4.1 is the following claim.

Claim 4.2. ([14]) For $\alpha=8$, every graph $H$ on at most $2 \alpha k$ vertices with minimum degree at least $\alpha$ contains a $k$-linked subgraph.

If one proves Claim 4.2 for any $3 \leqslant \alpha<8$, this would imply the strengthening of Theorem 4.1 with $\alpha$ in place of 8 . Using Theorem 1.1, we prove below an analog of Claim 4.2 with $\alpha=6$. This will make Theorem 4.1 work with 6 in place of 8 , i.e., will yield Theorem 1.3. The beginning of the proof is reminiscent of that for Claim 4.2, but for completeness, we present the full proof.

Lemma 4.3. For $\alpha=6$, every graph $H$ on at most $2 \alpha k$ vertices with minimum degree at least $\alpha k$ contains a k-linked subgraph.

Proof. Consider an $H$ satisfying the conditions of the lemma. If $H$ itself is not $k$-linked, then there is a set $X=\left\{s_{i}, t_{i}: 1 \leqslant i \leqslant k\right\} \subseteq V(H)$ such that there are no disjoint paths $P_{1}, \ldots, P_{k}$ such that each $P_{i}$ is an $s_{i}, t_{i}$-path and all $s_{i}$ and $t_{i}$ are distinct. Link as many as possible pairs $\left(s_{i}, t_{i}\right)$ by paths of length at most 6 and, subject to this, minimize the sum of the lengths of these paths. Suppose that $l_{1}$ pairs are not linked and the number of paths of length $i$ is $l_{i+1}, 1 \leqslant i \leqslant 6$. We may assume that $s_{1}$ and $t_{1}$ are not linked.

Let $S$ be the union of $X$ with vertex sets of all the paths of the linkage. Let $A=$ $N_{H}\left(s_{1}\right)-S$ and $B=N_{H}\left(t_{1}\right)-S$. By the choice, $A$ and $B$ are disjoint and are at distance at least 5 in $H-S$. Since the paths $P_{i}$ are chosen to be of the minimum total length, we have

$$
\begin{aligned}
\left|N_{H}(v) \cap V\left(P_{i}\right)\right| \leqslant 3 \quad & \forall v \in V(G)-S \quad \forall P_{i}, \\
\left|N_{H}(v) \cap\left\{s_{1}, t_{1}\right\}\right| \leqslant 1 \quad \forall v \in V(G)-S, &
\end{aligned}
$$

and

$$
\left|N_{H}\left(s_{1}\right) \cap N_{H}\left(t_{1}\right) \cap V\left(P_{i}\right)\right| \leqslant 3 \quad \forall i
$$

Claim 4.4. For each $v \in V(H)-S-A-B,|N(v) \cap(A \cup B)| \geqslant 2$ and either $N(v) \cap A=\emptyset$ or $N(v) \cap B=\emptyset$.

Proof. Suppose that $|N(v) \cap(A \cup B)| \leqslant 1$ for some $v \in V(H)-A-B$. By (4.1), (4.2), and (4.3), $\left|N_{H}(v)-S\right| \geqslant \delta(H)-3(k-1)-1$ and $|A|+|B| \geqslant 2 \delta(H)-|S|-3 k$, we have

$$
|V(H)| \geqslant|A|+|B|+|S|+\left(1+\operatorname{deg}_{H-S}(v)\right)-1 \geqslant 2+3 \delta(H)-6 k>12 k \geqslant|V(H)|,
$$

a contradiction. If both $N(v) \cap A \neq \emptyset$ and $N(v) \cap B \neq \emptyset$, then there is an $s_{1}, t_{1}$-path of length 4 outside $S$, a contradiction. 
By Claim 4.4, every vertex in $V(H)-S$ has distance at most two from either $s_{1}$ or $t_{1}$, but not both. Therefore, $H-S$ is the union of $H_{1}=H[A \cup N(A)-S]$ and $H_{2}=$ $H[B \cup N(B)-S]$, and there are no edges connecting $V\left(H_{1}\right)$ with $V\left(H_{2}\right)$. Assume that $\left|V\left(H_{1}\right)\right| \leqslant\left|V\left(H_{2}\right)\right|$.

Observe that $\delta\left(H_{1}\right) \geqslant \delta(H)-3\left(k-l_{1}\right)-l_{1} \geqslant 6 k-3 k+2 l_{1}=3 k+2 l_{1}$ and $\left|V\left(H_{1}\right)\right| \geqslant$ $1+\delta\left(H_{1}\right)>3 k$. If $\left|V\left(H_{1}\right)\right| \leqslant 4 k$, then $3 k+2 l_{1} \geqslant \frac{\left|V\left(H_{1}\right)\right|+5 k}{3}$, and by Theorem 1.1, $H_{1}$ is $k$-linked. This proves the lemma when $\left|V\left(H_{1}\right)\right| \leqslant 4 k$.

Now consider the case $\left|V\left(H_{1}\right)\right|,\left|V\left(H_{2}\right)\right| \geqslant 4 k+1$. If $\delta\left(H_{1}\right) \geqslant\left(\left|V\left(H_{1}\right)\right|-3\right) / 2+k$ or $\delta\left(H_{2}\right) \geqslant\left(\left|V\left(H_{2}\right)\right|-3\right) / 2+k$, then by Theorem 1.1 , either $H_{1}$ or $H_{2}$ is $k$-linked. Thus we may assume that there exist $v_{1} \in H_{1}$ and $v_{2} \in H_{2}$ such that $\operatorname{deg}_{H_{1}}\left(v_{1}\right) \leqslant\left|V\left(H_{1}\right)\right| / 2+k-2$ and $\operatorname{deg}_{H_{2}}\left(v_{2}\right) \leqslant\left|V\left(H_{2}\right)\right|+k-2$. Hence,

$$
\operatorname{deg}_{H_{1}}\left(v_{1}\right)+\operatorname{deg}_{H_{2}}\left(v_{2}\right) \leqslant\left(\left|V\left(H_{1}\right)\right|+\left|V\left(H_{2}\right)\right|\right) / 2+2 k-4,
$$

that is,

$$
\operatorname{deg}_{H}\left(v_{1}\right)+\operatorname{deg}_{H}\left(v_{2}\right)-\left(\left|N_{H}\left(v_{1}\right) \cap S\right|+\left|N_{H}\left(v_{2}\right) \cap S\right|\right) \leqslant(|V(H)|-|S|) / 2+2 k-4 .
$$

Therefore

$$
\begin{aligned}
\left|N_{H}\left(v_{1}\right) \cap S\right|+\left|N_{H}\left(v_{2}\right) \cap S\right| & \geqslant 2 \delta(H)-|V(H)| / 2+|S| / 2-2 k+4 \\
& \geqslant 12 k-6 k+|S| / 2-2 k+4 \geqslant 4 k+4+|S| / 2 .
\end{aligned}
$$

Claim 4.5. $\left|N\left(v_{1}\right) \cap S\right|+\left|N\left(v_{2}\right) \cap S\right| \leqslant 4 l_{1}+4 l_{2}+5 l_{3}+6\left(l_{4}+l_{5}+l_{6}+l_{7}\right)$.

Proof. It is enough to prove that $v_{1}$ and $v_{2}$ together have at most:

(a) 4 neighbours (counted with multiplicities) on each $P_{i}$ of length 1 and each unlinked pair in $S$,

(b) 5 neighbours on each $P_{i}$ of length 2 ,

(c) 6 neighbours on each $P_{i}$ of length at least 3 .

Statement (a) is evident and (c) follows from (4.1). To prove (b), suppose that each of $v_{1}$ and $v_{2}$ has exactly 3 neighbours in a path $P_{i}=\left(s_{i}, w, t_{i}\right)$. Since $|S| \leqslant|V(H)|-$ $\left|V\left(H_{1}\right)\right|-\left|V\left(H_{2}\right)\right| \leqslant 12 k-2(4 k+1)=4 k-2$, every $v \in V(H)$ has at least $6 k-|S| \geqslant 2 k+$ 2 neighbours outside $S$. Thus we may assume that $w u \in E(G)$ for some $u \in V\left(H_{1}\right), u \neq v_{1}$.

Since no $u \in V\left(H_{1}\right)+s_{1}$ is adjacent to any $w \in V\left(H_{2}\right)+t_{1}$, each distinct pair $u_{1}, u_{2} \in$ $V\left(H_{1}\right)+s_{1}$ has at least $6 k+6 k-(8 k-2)=4 k+2 \geqslant|S|+4$ common neighbours. Similarly, each distinct pair $w_{1}, w_{2} \in V\left(H_{2}\right)+t_{1}$ has at least $|S|+4$ common neighbours. Thus, the graph $H-\left(S-s_{1}-t_{1}\right)$ contains an $s_{1}$, u-path $Q_{1}$ of length at most 2 avoiding $v_{1}$ and a $t_{1}, v_{2}$-path $Q_{2}$ of length at most 2 . Then we replace $P_{i}$ by $s_{i} v_{1} t_{i}$ and add the path $s_{1} Q_{1} u w v_{2} Q_{2} t_{1}$ of length at most 6 . This contradicts the maximality of the linkage. 
Now by Claim 4.5 we have

$$
\begin{aligned}
\left|N\left(v_{1}\right) \cap S\right|+\left|N\left(v_{2}\right) \cap S\right| & \leqslant 4 l_{1}+4 l_{2}+5 l_{3}+6\left(l_{4}+l_{5}+l_{6}+l_{7}\right) \\
& =4 \sum_{i=1}^{7} l_{i}+\left(l_{3}+2 l_{4}+2 l_{5}+2 l_{6}+2 l_{7}\right) \\
& =4 k+\left(l_{3}+2 l_{4}+2 l_{5}+2 l_{6}+2 l_{7}\right) \leqslant 4 k+|S| / 2,
\end{aligned}
$$

which contradicts (4.4).

As we have mentioned above, proving Lemma 4.3 for smaller $\alpha$ would yield the corresponding improvement for $f(k)$.

\section{Acknowledgement}

We thank a referee for helpful comments.

\section{References}

[1] Bollobás, B. and Thomason, A. (1996) Highly linked graphs. Combinatorica 16 313-320.

[2] Dirac, G. (1952) Some theorems on abstract graphs. Proc. London Math. Soc. 2 69-81.

[3] Egawa, Y., Faudree, R. J., Győri, E., Ishigami, Y., Schelp, R. H. and Wang, H. (2000) Vertexdisjoint cycles containing specified edges. Graphs Combin. 16 81-92.

[4] Faudree, J., Faudree, R., Gould, R., Jacobson, M. and Lesniak, L. (2000) On $k$-ordered graphs. J. Graph Theory 35 69-82.

[5] Gould, R. J. (2003) Advances on the Hamiltonian problem: A survey. Graphs Combin. 19 7-52.

[6] Gould, R. J., Kostochka, A. V., Lesniak, L., Schiermeyer, I. and Saito, A. (2003) Degree conditions for $k$-ordered Hamiltonian graphs. J. Graph Theory 42 199-210.

[7] Jung, H. A. (1970) Verallgemeinerung des $n$-fachen Zusammenhangs für Graphen. Math. Annalen 187 95-103.

[8] Kierstead, H. A., Sárközy, G. and Selkow, S. (1999) On k-ordered Hamiltonian graphs. J. Graph Theory 32 17-25.

[9] Larman, D. G. and Mani, P. (1974) On the existence of certain configurations within graphs and the 1-skeletons of polytopes. Proc. London Math. Soc. 20 144-160.

[10] Mader, W. (1967) Homomorphieeigenschaften und mittlere Kantendichte von Graphen. Math. Annalen 174 265-268.

[11] Ng, L. and Schultz, M. (1997) k-ordered Hamiltonian graphs. J. Graph Theory 2 45-57.

[12] Ore, O. (1960) Note on Hamilton circuits. Amer. Math. Monthly 6755.

[13] Robertson, N. and Seymour, P. D. (1995) Graph minors XIII: The disjoint path problem. J. Combin. Theory Ser. B, 63 65-110.

[14] Thomas, R. and Wollan, P. (2003) An improved linear edge bound for graph linkage. Submitted. 